CERIAS Tech Report 2001-72

An overview of multimedia content protection in consumer electronics devices

by Ahmet M. Eskicioglu* and Edward J. Delp

Center for Education and Research in

Information Assurance and Security,

Purdue University, West Lafayette, IN 47907-2086 


\title{
An overview of multimedia content protection in consumer electronics devices
}

\author{
Ahmet M. Eskicioglu* and Edward J. Delp \\ *Thomson Consumer Electronics \\ Corporate Research \\ $101 \mathrm{~W} . \mathbf{1 0 3}^{\text {rd }}$ Street \\ Indianapolis, Indiana 46290-1102 \\ USA \\ *Video and Image Processing Laboratory (VIPER) \\ School of Electrical and Computer Engineering \\ Purdue University \\ West Lafayette, Indiana 47907-1285 \\ USA
}

\begin{abstract}
A digital home network is a cluster of digital audio/visual (A/V) devices including set-top boxes, TVs, VCRs, DVD players, and general-purpose computing devices such as personal computers. The network may receive copyrighted digital multimedia content from a number of sources. This content may be broadcast via satellite or terrestrial systems, transmitted by cable operators, or made available as prepackaged media (e.g., a digital tape or a digital video disc). Before releasing their content for distribution, the content owners may require protection by specifying access conditions. Once the content is delivered to the consumer, it moves across home the network until it reaches its destination where it is stored or displayed. A copy protection system is needed to prevent unauthorized access to bit streams in transmission from one $\mathrm{A} / \mathrm{V}$ device to another or while it is in storage on magnetic or optical media. Recently, two fundamental groups of technologies, encryption and watermarking, have been identified for protecting copyrighted digital multimedia content. This paper is an overview of the work done for protecting content owners' investment in intellectual property.
\end{abstract}

Keywords: multimedia, copy protection, cryptography, watermarking, consumer electronics, digital television, digital video disc, digital video cassette, home networks.

\section{INTRODUCTION}

In the entertainment world, original multimedia content (e.g., text, audio, video and still images) is made available for consumers through a variety of channels. Modern distribution systems allow the delivery of content to millions of households every day. Although legal institutions exist for protecting intellectual property (trademarks and patents) owned by content creators, complimentary technical measures are needed to sustain financial returns and to ensure incentives for new creations.

In order to see the increasing importance of protecting copyrighted content, one should understand an essential difference between old and new technologies for distribution and storage. Prior to the development of digital technologies, content was created, distributed, stored and displayed by analog means. The popular video cassette recorders (VCRs) of the 1980's introduced a revolutionary way of viewing A/V content, but ironically allowed unauthorized copying, risking the investments made in intellectual property. An inherent

\footnotetext{
* Address all correspondence to A. M. Eskicioglu at eskicioglua@tce.com. E. J. Delp can be reached at ace@ecn.purdue.edu. Portions of this work done by EJD were supported by the sponsors of the Center for Education and Research in Information Assurance and Security.
} 
characteristics of analog recording, however, prevented piracy efforts to reach alarming proportions. If a taped content is copied on a VCR, the visual quality of the new, i.e., the first-generation, copy is reduced. Further generational copies result in noticeably less quality, decreasing the commercial value of the content. Today, reasonably efficient analog copy protection methods exist, and have recently been made mandatory, in consumer electronics devices to further discourage illegal analog copying.

With the advent of digital technologies, new tools have emerged for making perfect copies of the original content. A quick review of digital representation of data will reveal why generational copies do not lose their quality. A text, an image or a video is represented as a stream of bits (0's and 1's). This representation can be conveniently stored on magnetic or optical media. Since digital recording is a process whereby each bit in the source stream is read and copied to the new medium, an exact replica of the content is obtained. Such a capability becomes even more threatening with the every increasing availability of Internet, an immense and boundless digital distribution mechanism. Protection of digital multimedia content therefore appears to be a new and crucial problem for which immediate solutions are needed.

Three major industries have a great interest in this problem:

1. Motion picture industry

2. Consumer electronics (CE) industry

3. Information technology (IT) industry

The content owners are the motion picture studios. Their content (movies) is displayed or recorded on devices manufactured by consumer electronics companies. The information technology industry manufactures general purpose computing devices, such a personal computers, which can also be used to display and store content.

Research conducted by the CE and IT industries has revealed two promising groups of technologies. Encryption-based technologies transform content into unintelligible or unviewable form. This transformation, being reversible in nature, allows perfect recovery of content. Both symmetric and public key ciphers are commonly used for content security and authentication (see Section 2). Technologies based on watermarking serve several purposes: identification of the content origin, tracing illegal copies and disabling unauthorized access to content.

With constant feedback provided by the content owners, the CE and IT industries have been developing solutions in specific areas. The Content Scramble System (CSS), for example, provides protection for content recorded on DVD-ROM discs (see Section 4). Other systems are proposed for securing the IEEE 1394 interface, and preventing unauthorized copies on recordable DVDs (DVD-R/RW/RAM).

An alternative approach is to develop global architectures based on removable security devices. Such architectures are considered extensions of conditional access systems, restricting viewing when the consumer does not have the correct entitlements. The National Renewable Security Standard (NRSS) provides a means for separating the security functionality from navigational devices (see Section 5).

The recording industry is another major player in the copy protection arena who has chosen a separate path to develop a solution for musical content. The recent launch of the Secure Digital Music Initiative (SDMI) is a indication of the strong need for secure distribution of music (see Section 8).

This paper highlights recent developments in protecting copyrighted multimedia content. The exact details of the copy protection systems will be omitted throughout the presentation due to security issues and for the protection of intellectual property. 


\section{BASIC CONCEPTS AND DEFINITIONS IN CRYPTOGRAPHY AND WATERMARKING}

To have a better understanding of the impact of protection methods on consumer electronics devices, we will start with a summary of basic concepts and definitions in cryptography and watermarking.

\subsection{Cryptography}

Cryptography $1,2,3,4,5$ deals with the concealment and protection of digital information. The study of cryptographic techniques is more than 400 years old. Shannon's 1949 paper $^{6}$ that connected cryptographic techniques with digital communication theory is thought by many to be the beginning of "modern" cryptography ${ }^{7}$.

A cryptographic system consists of five elements: A plaintext message space, a ciphertext message space, a key space, a family of enciphering transformations, and a family of deciphering transformations. In modern cryptosystems, the enciphering and deciphering transformations are public, only the keys need to be kept secret. Cryptanalysis is the science and study of "breaking" or attacking ciphers.

Ciphers can be classified according to two important criteria: (1) symmetric vs. asymmetric and (2) stream vs. block.

In a symmetric key cipher, enciphering and deciphering keys are the same or can easily be determined from each other. Asymmetric key systems (public key systems) differ in such a way that at least one key is computationally infeasible to determine from the other. The key used for encryption is publicly available, while the corresponding decryption key should be kept confidential all the time. ${ }^{8,9}$

A stream cipher breaks the message $M$ into successive characters or bits $m_{1}, m_{2}, m_{3}, \ldots$, and enciphers each $m_{i}$ with the ith element $k_{i}$ of a key stream $K=k_{1} k_{2} k_{3}, \ldots$ A block cipher breaks the message $M$ into successive blocks $M_{1}, M_{2}, M_{3}, \ldots$, and enciphers each $M_{i}$ with the same key $K$.

An example of symmetric and asymmetric key ciphers is shown is Figure 1. When the two parties A and B want to communicate securely, each approach introduces key management problems. In case (a), both parties need to have a copy of the symmetric key, the distribution of which is a non-trivial problem. The problem with case (b) is the authentication of the public key that is used for encryption; A needs assurance that it has the public key that actually belongs to $\mathrm{B}$.

Using public key cryptographic techniques, one can provide assurance about the integrity or reliability of a public key or other types of data. This is usually referred to as authentication. ${ }^{4}$ There are two types of authentication protocols. In message authentication, a party is corroborated as the original source of specified data created at some time in the past. In entity authentication, one party is assured of the identity of a second party involved in a protocol, and that the second party has actually participated.

A digital signature, ${ }^{4}$ which associates a message with some originating entity, can be constructed with public key systems. Each digital signature scheme includes a signature generation algorithm and a signature verification algorithm. A public key certificate ${ }^{5}$ is a digitally signed message consisting of two parts which can be used to authenticate a public key. The "data part" includes the public key that is being authenticated, as well as other information such as the issuer, the owner, and the validity period of the public key. The "signature part" is the signature of the data part generated by the issuer of the certificate. 


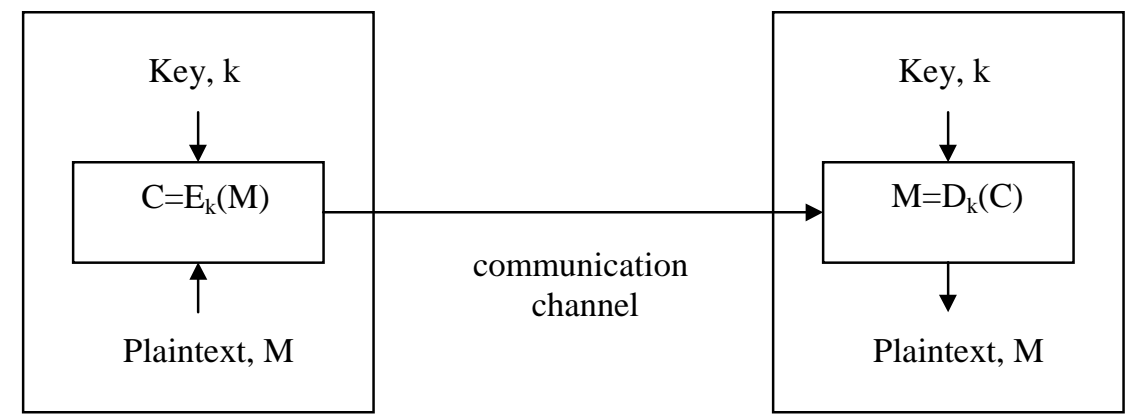

A

B

(a)

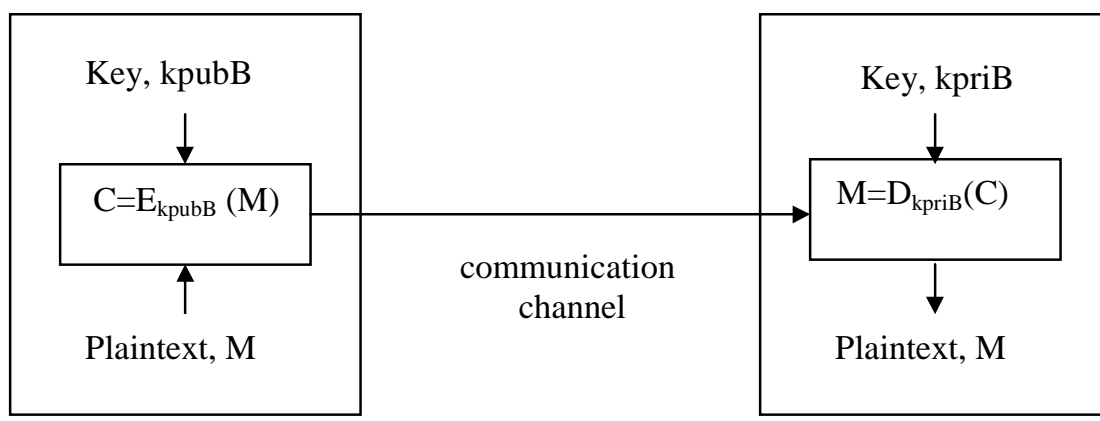

A

B

(b)

Figure 1. Encryption: (a) symmetric key, (b) asymmetric key

\subsection{Watermarking and data hiding}

Watermarking ${ }^{10,11}$ is the process of embedding data (or controlled distortion) into a multimedia element such as image, audio and video. This embedded information, known as the watermark, can later be extracted from the multimedia and used for security purposes. ${ }^{12}$ In multimedia applications, the watermark should be invisible or inaudible to the human observer (visible watermarking techniques do exist). ${ }^{13}$ A watermarking algorithm consists of the watermark structure, an embedding algorithm and an extraction or detection algorithm. Watermarks can be embedded into multimedia directly (e.g., the time domain) or after the multimedia element has been transformed (e.g., the discrete cosine transform). ${ }^{14}$ Performance issues include robustness to attack (attempts to remove the watermark), capacity (how bits can be hidden in the multimedia) and how transparent is the watermark under normal viewing or listening conditions. There has been a tremendous amount of work done in watermarking in the past 6 years. ${ }^{11}$

Typical uses of watermarks include identification of the origin of content, tracing illegally distributed copies, and disabling unauthorized access to content. A mature robust watermarking technology should be resistant to many types of attacks and normal $\mathrm{A} / \mathrm{V}$ processes such as noise, filtering, resampling, cropping, data compression, and A-to-D and D-to-A conversions. 


\subsection{Multilayer protection by encryption and watermarking}

Encryption or watermark based technologies can be independently used for protecting multimedia content. However, it is possible to implement both in the same application, providing a two-layer protection. As shown in Figure 2, the content may have been watermarked immediately after creation. The sending party encrypts the watermarked content to provide the second layer of protection. At the receiving end, the stream is decrypted before watermark detection takes place.

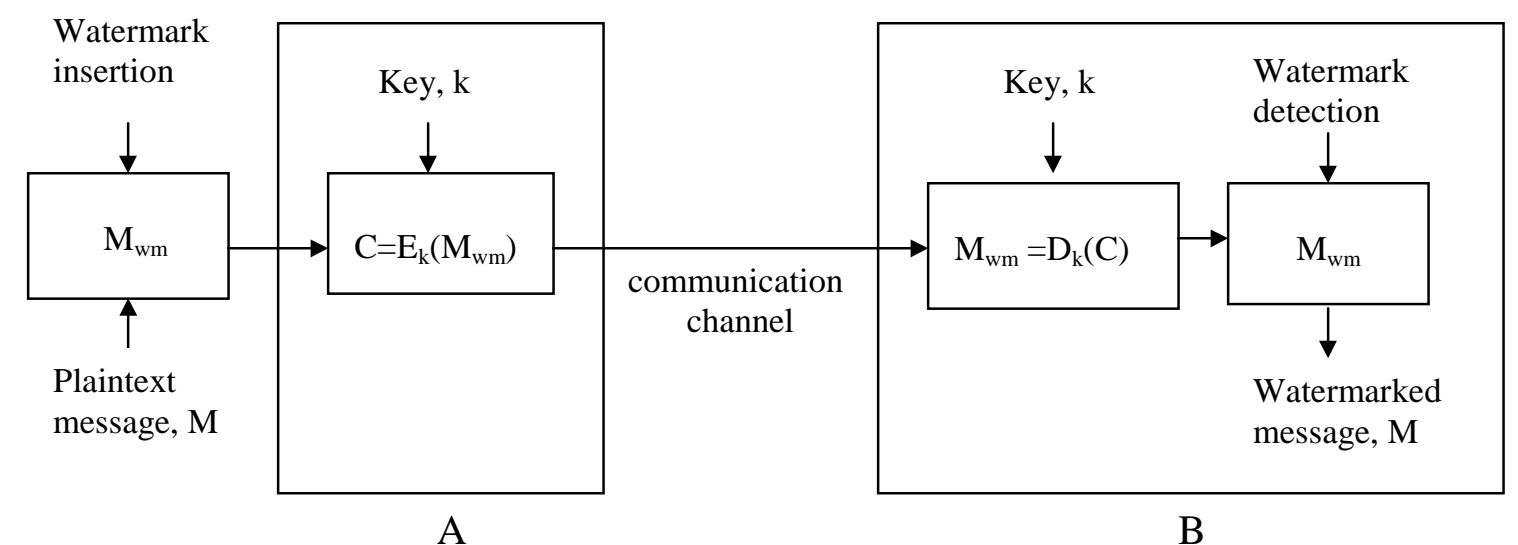

Figure 2. Two-layer protection

\section{THE BEGINNINGS}

The work on copy protection started almost four years ago. At the beginning of 1996, a bill was drafted as a result of collaboration between consumer electronics companies and content owners. The "Video Home Recording Act of 1996" was intended to amend title 17, United States Code, to "govern the importation, manufacture and distribution of digital motion picture recording and related services, to prohibit certain copyrighted infringement actions, and for other purposes." One section of the Act was a technical reference document for establishing the standards and specifications for implementing technological management of consumer copying of linear motion pictures. Before the bill was actually submitted to the US Congress, the three industries (CE, IT and motion picture) wanted to resolve all outstanding issues, and agreed to create a forum for discussion.

The forum gave birth to a plenary group which was comprised of both technical and policy representatives of the member companies of the MPAA ${ }^{\dagger}, \mathrm{CEMA}^{\ddagger}, \mathrm{BSA}^{\S}$, ITIC $^{* * *}$, and RIAA. ${ }^{\dagger \dagger}$ Each expertise group (technical and policy) was assigned a specific task that was completed in late June of 1996. The findings were presented in two reports on June 21, 1996. The report of the policy group summarized the exploratory discussions regarding the concepts of anti-circumvention in conjunction with the introduction of digital video technologies, and the key policy considerations to be weighed in making decisions about specific technical and legislative proposals. Focusing on technical issues, the other group identified and evaluated the technical approaches to protect content in analog or digital form, delivered by direct electronic transmission or prerecorded media. After these presentations, the technical group, now known as the Copy Protection Technical Working Group ${ }^{15}$

\footnotetext{
$\dagger$ Motion Picture Association of America (http://www.mpaa.org).

¥ Consumer Electronics Manufacturing Association (now known as the Consumer Electronics Association, http://www.ce.org).

$\S$ Business Software Alliance (http://www.bsa.org).

** Information Technology Industry Council (http://www.itic.org).

t† Recording Industry Association of America (http://www.riaa.org/).
} 
(CPTWG), continued discussing copy protection problems. It is still active today, having monthly meetings to discuss the current issues.

In the past three years, CPTWG formed working groups to focus on specific problems. ${ }^{16}$ Two of the most active groups were the Digital Transmission Discussion Group (DTDG) and the Data Hiding Subgroup (DHSG).

The DTDG was created on October 3, 1996. Its scope was to define a data protection system (DPS) that can be used to protect digital audio/video transmitted on the IEEE 1394-1995 high performance serial bus. ${ }^{17}$ The architecture developed for DPS had three layers:

1. Copy Control Information (CCI) Layer - a means of carrying information along with the copyrighted content that expresses the intentions of the copyright holder with regard to the conditions under which an end consumer is authorized to make a copy.

2. Device Authentication and Key Exchange Layer - a means of a compliant device to establish the authenticity of another device prior to exchanging copyrighted content, and also to generate the keys for data encryption.

3. Data Encryption Layer - a means of encrypting the copyrighted content when it is transmitted from one compliant device to another compliant device in digital form.

The DTDG issued a Call for Proposals on March 11, 1997, and published its "Review and Findings" report ${ }^{18}$ summarizing the technical features of the submitted proposals. After completing its task, the DTDG closed in February 1998. Five of the proposals included in the DTDG report later merged to form the 5C group. ${ }^{19}$

The DHSG was created on May 6, 1997. Its scope was to define a data hiding system that can be used to mark video content for the purposes of identifying marked material and preventing unauthorized recording/playback. The Call for Proposals issued on July 1, 1997 identified a set of essential and desirable requirements for the system. The Interim Report ${ }^{20}$ published by DHSG included the performance of seven proposals during visibility and survivability tests.

Because of prolonged discussions, the "Video Home Recording Act of 1996" could not be submitted to the US Congress.

\section{DVD PROTECTION}

\subsection{DVD Video}

The first problem addressed CPTWG was the protection of content on DVD Video discs developed by the DVD Consortium (now known as the DVD Forum). The DVD Consortium was started as an ad hoc group in December 1995 to promote a single format for a large capacity disc, now known as DVD. The founding members were Hitachi, MEI, Mitsubishi, Philips, Pioneer, Sony, Thomson, Time Warner, Toshiba and Victor. With over 100 member companies today, the DVD Forum defines the specifications for DVDs. Currently, it has eight working groups: WG1: DVD Video, WG2: DVD-ROM Physical Format, WG3: DVD File System, WG4: DVD Audio, WG5: DVD-RAM Physical Format, WG6: DVD-R/RW Physical Format, WG9: DVD Copy Protection, WG10: DVD Professional Use.

Several proposals were studied by the DVD Forum and CPTWG. After much discussion and critical review, the DVD Forum recommended the proposal developed by MEI and Toshiba to the relevant industries. Known as the Content Scramble System (CSS), the system consists of a private scrambling system with multi-layer key management. Scrambling takes place at the disc manufacturing location before the discs are pressed. As shown in Figure 3, the CSS-protected content is descrambled during playback on a DVD player. The CSS has been very much in the news lately because a group of computer hackers have successfully attacked CSS ${ }^{21}$ Note that the first generation players are allowed to have NTSC (analog) output only. An analog protection system (APS) developed by Macrovision results in degradation in unauthorized copies made on VHS recorders. 


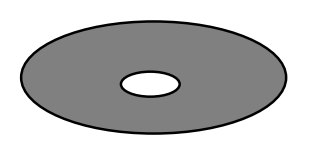

CSS-scrambled content

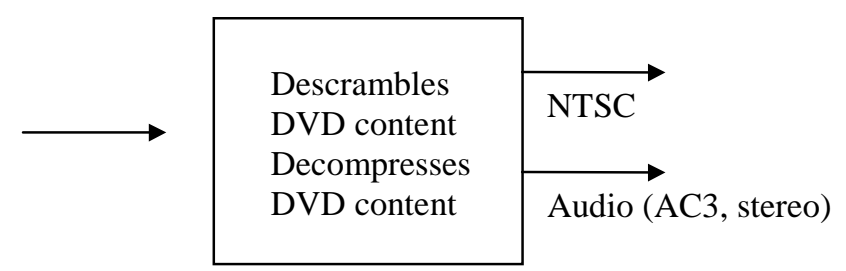

DVD player

Figure 3. CSS on a DVD player.

Figure 4 shows the additional element needed in CSS for implementation on a PC system. The DVD drive and the PC participate in mutual authentication before the scrambled content is sent to the descrambler. This allows each party to check if the other participant is authorized to handle CSS scrambled content.

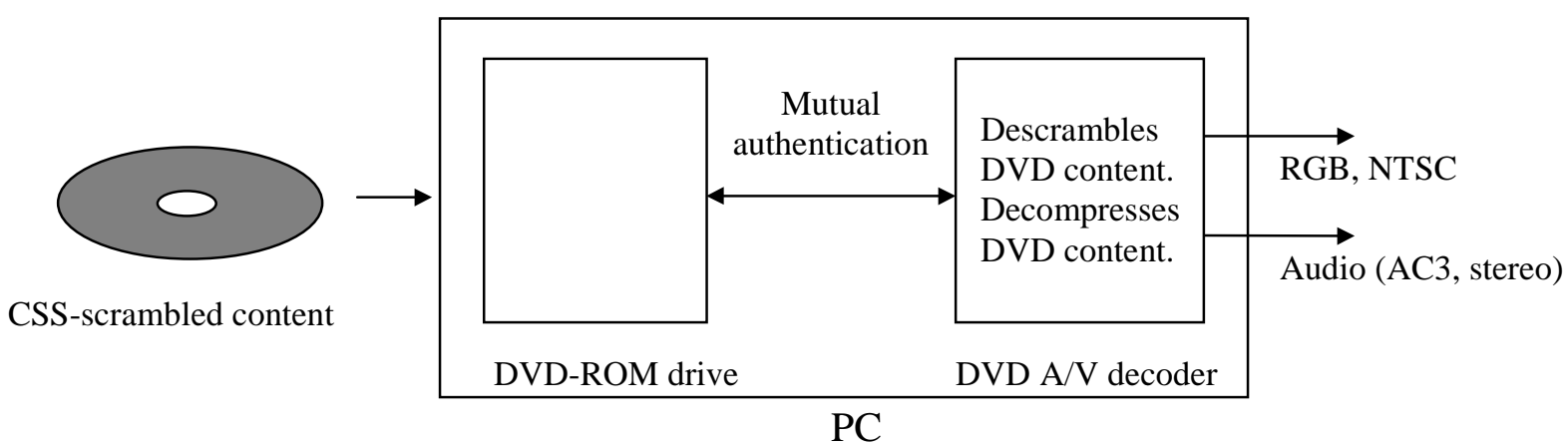

Figure 4. CSS on a PC system.

The DVD Copy Control Association (CCA) is the entity created to license the CSS technology. The CSS Specifications are provided for each licensee to have access to the appropriate information for implementation. It includes two sections: Procedural and technical. The procedural section provide the terms and conditions of the use of CSS specifications, while the technical section, designated specifically for particular membership categories, describes the system components.

\subsection{DVD Audio}

The experience gained in DVD Video protection has helped considerably in determining an architecture for protecting prerecorded DVD Audio discs. An important factor taken into consideration for this architecture was the existence of the compact disc (CD), the first generation of digital audio format. It was argued that since a large population of $\mathrm{CD}$ players were still in the field, the consumers would most likely desire to have copies on recordable $\mathrm{CD}$ media during the transition period.

With input from the major recording studios, four companies (IBM, Intel, MEI and Toshiba) proposed a framework where watermarking and encryption are the primary technologies for preventing unauthorized playback or recording. The DVD Audio copy protection framework, which allows personal copies when authorized by content owners, is defined by the following rules: 
- Devices need to have a license to descramble and to detect watermarks.

- Copying is limited to one per recorder unless more copies are authorized.

- Authorized copies must be scrambled to prevent further copies (Unprotected copies are allowed on legacy media with restricted sound quality).

- Copying for personal use is allowed at CD-Audio sound quality (Additional copies with different characteristics may be authorized by selecting different values of CCI parameters).

- The CCI parameter values must be sent securely to a licensed recording device together with scrambled content.

- Content in unscrambled digital or analog form can be sent to a licensed recorder with specific values of $\mathrm{C}$ and $\mathrm{Q}$ parameters (see below) embedded in the audio watermark.

- All outputs of DVD-Audio content except IEC-958 and analog from licensed DVD devices must be scrambled by an approved system.

- The robustness of implementation must be similar to that of CSS.

The CCI parameters, namely C, Q, R and T, allow the content owners to specify on a track by track basis the conditions for copying. Their definition and a set of permissible values that have to be supported by playback and recording devices are given in Table 1.

\begin{tabular}{|c|c|}
\hline \multirow[b]{2}{*}{ C } & Specifies the number of, or other conditions for, copies authorized per recorder \\
\hline & $\begin{array}{l}\mathrm{N}: \text { Number of copies } \mathrm{N}=1 \text { (default value) } \\
\text { One generation } \\
\text { No more copies } \\
\text { No copy control }\end{array}$ \\
\hline \multirow[b]{2}{*}{ Q } & Specifies the maximum sound quality of the permitted recording \\
\hline & $\begin{array}{l}\text { CD-Audio quality (default value) } \\
\text { 2-channel full quality } \\
\text { Multi-channel full quality }\end{array}$ \\
\hline \multirow[b]{2}{*}{$\mathbf{R}$} & Indicates the authorization status for copies of each element of related content \\
\hline & $\begin{array}{l}\text { Authorized } \\
\text { Unauthorized }\end{array}$ \\
\hline \multirow[b]{2}{*}{$\mathbf{T}$} & Provides optional access control parameters \\
\hline & $\begin{array}{l}\text { Values downloaded to the DVD Audio player from the Internet may override the } \\
\text { CCI on the Audio disc }\end{array}$ \\
\hline
\end{tabular}

Table 1. Permissible CCI parameter settings.

The copy protection framework needs the support of three systems:

1. A scrambling system for prerecorded DVD Audio discs

2. A watermarking system for embedding CCI in the content

3. A system for creating secure authorized copies

Work is in progress to develop these component technologies. Specifications of Copy Protection for Prerecorded Media (CPPM), an audio watermarking system, and CPRM (for authorized copies, see Section 4.3) are being finalized.

\subsection{Recordable DVDs (RAM/R/RW)}

The DVD Forum WG9, the working group addressing copy protection, is in the process of determining the components of the security architecture for recordables DVDs. A summary of the work is given in Table 2. 


\begin{tabular}{|l|l|}
\hline Component & Decision \\
\hline Disc type recognition & Will be implemented \\
\hline CCI & Will be implemented \\
\hline Watermark & Will be implemented \\
\hline Secure transmission & Will be implemented \\
\hline Encryption & Will be implemented \\
\hline Compliance mark & Under discussion \\
\hline Ticket & Under discussion - used by a particular watermarking technology \\
\hline Authentication & Being studied for the PC environment \\
\hline Unique disc ID & Under discussion \\
\hline
\end{tabular}

Table 2. Components of the copy protection architecture for recordable DVDs

Developed by IBM, Intel, MEI, and Toshiba, the proposal known as Content Protection for Recordable Media (CPRM) provides some of the components given in Table 2. Although the CPRM technology presently addresses only one DVD physical format (DVD-RAM 4.7 GB) and one application format (video recording), other physical and application formats will be considered in future revisions. The principal elements of CPRM include a private key management system and disc type recognition.

As noted earlier, the first generation DVD players were limited to have analog output only. There was not an immediate need to protect a digital stream leaving a DVD player. In home networks, however, there will be several devices (including newer generation of DVD players) with digital interfaces that need to be protected.

\subsection{IEEE 1394 interface protection}

The Digital Transmission Content Protection (DTCP) specification was jointly developed by Hitachi, Intel, MEI, Sony and Toshiba. ${ }^{19}$ It defines a cryptographic protocol for protecting audio/video entertainment content from unauthorized copying, intercepting, and tampering as it traverses digital transmission mechanisms such as a serial bus that conforms to the IEEE 1394-1995 standard. The use of this specification, and the intellectual property and cryptographic information required to implement it, are subject of a license. The Digital Transmission Licensing Administrator (DTLA) is responsible for establishing and administering the system described in the specification. The DTCP system addresses the three DTDG layers in the following way:

Copy Control Information (CCI) layer:

The CCI can be carried in two ways: Encryption Mode Indicator (EMI) and embedded CCI. The most significant bits of the synch field of the isochronous packet header are used for encoding the (EMI) bits as follows:
11: copy-never
10: copy-one-generation
01: no-more-copies
00: copy-free

CCI can also embedded in the content stream (to be recognized by format-cognizant devices).

Authentication and key exchange layer:

Two authentication levels are provided: Full and restricted. Full authentication can be used with all types of content protected by the system. Restricted authentication is applicable for the protection of "copy-onegeneration" and "no-more-copies" content only. 
Content encryption layer:

The $\mathrm{M}^{4}$ cipher is defined as the baseline cipher, i.e., the cipher that must be supported by all compliant devices for interoperability. It is a symmetric-key block cipher based on permutation and substitution. Other ciphers such as the Data Encryption Standard (DES) ${ }^{4}$ and Modified Blowfish ${ }^{4}$ can also be supported.

As a result of a request from the content providers, a fourth layer has been added to allow system renewability.

System renewability layer:

Since the security of the DTCP system relies on the secrets embedded in devices, it is not possible to renew the system by replacing the secrets. Renewability is therefore implemented using the concept of revocation. A Certificate Revocation List (CRL) is a list of device IDs identifying the devices with compromised security. ${ }^{19}$ The DTLA generates and distributes such lists in System Renewability Messages (SRMs). Devices that support full authentication receive and store SRMs for device revocation. SRMs are updated via new content or new services in a number of ways. Some alternatives are other compliant devices with newer lists, prerecorded content media, and compliant devices with external communication capability (e.g. Internet, cable or satellite connections).

\subsection{Historical look at DVD protection}

Figure 5 depicts three systems needed to protect DVD content in home networks. The CSS scrambles the content before it is recorded on a DVD ROM. The first generation DVD player outputs an NTSC signal after decryption and MPEG decoding. If the DVD player has a IEEE 1394 interface, the output should be protected by a second system (labeled X in Figure 5, e.g., 5C) that performs re-encryption. Being a compliant device, the receiving unit (e.g., a digital television) has the descrambling engine and the keys for recovering the video signal. The third system (labeled Y in Figure 5, e.g., CPRM) is needed for protecting the DVD content that was initially encrypted by CSS, and re-encrypted by X for transmission across the 1394 interface.

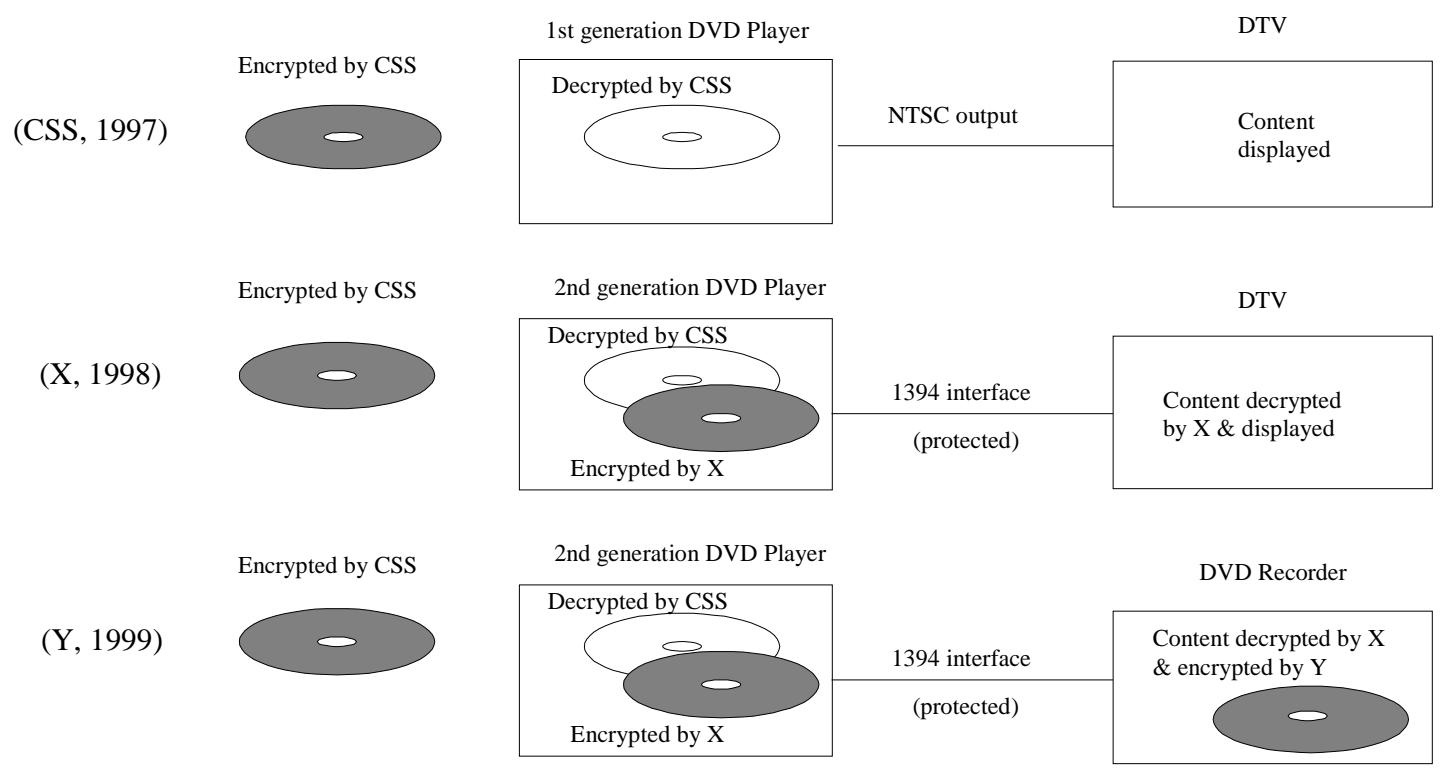

Figure 5. Three copy protection systems for DVD protection 


\subsection{Watermarking}

The CSS license includes the future use of a video watermarking technology for playback and recording control. The DVD CCA therefore needs to choose a watermarking technology as part of the license. The Watermark Review Panel (WaRP) was formed in December 1998 to assist CCA in evaluating the proposals. It had ten members representing the CE, IT and motion picture industries.

The seven video watermarking proposals submitted to DHSG were merged to form two groups: Galaxy (Hitachi, IBM, NEC, Pioneer and Sony) and Millennium (Digimarc, Macrovision and Philips). ${ }^{15}$ The key criteria used in testing these two candidates in the summer of 1999 were visibility, survivability, false positive rate, piracy, cost generational control for one copy, and licensing terms and conditions. According to the recent reports presented at the CPTWG meetings, Galaxy and Millennium performed similarly in the tests. A major architectural difference between the two is the scheme used for generational control. ${ }^{16}$ Galaxy inserts a new watermark in the copy, whereas Millennium processes a "ticket" (auxiliary data) attached to the content. The DVD CCA is expected to make a decision in the near future.

\section{CEMA AND COPY PROTECTION}

\subsection{National Renewable Security Standard (NRSS)}

The NRSS architecture was developed by CEMA partly in response to the Telecommunications Act of 1996. It provides a means for renewable security to be employed with digital consumer electronics devices such as digital television receivers and digital VCRs. Renewable security encompasses upgradeable, extensible, removable and replaceable security, allowing the security functionality to be separated from navigational devices. Simply stated, NRSS allows for the security system to be replaced if it has been hacked. This will be accomplished by "smart card" devices connected to consumer electronic devices.

The NRSS provides two physical designs, known as Part A and Part B. Part A defines a removable and renewable security element that is an extension of the ISO-7816 standard. ${ }^{22}$ Part B defines a removable and renewable security element based on the PCMCIA ("PC Card") form factor. The common attributes allow either an NRSS-A or NRSS-B device to provide security for applications involving pay and subscription cable or satellite television services, telephony, and all forms of electronic commerce.

The main differences between NRSS-A and NRSS-B devices are the range of capabilities and the capacity for extension. The NRSS-A interface is limited to 8 electrical contacts using serial communication, whereas the NRSS-B interface uses 68 electrical contacts and parallel communication. In general, the NRSS-A device could be smaller and less costly, while the NRSS-B device could be more robust and extensible.

\subsection{Interface protection}

CEMA has standardized four interfaces for device interconnection: IEEE 1394 interface, RF Remod interface, NRSS interface, and analog component video interface.

1. EIA-775: This standard ${ }^{23}$ defines a specification for a baseband digital interface to a digital television. It is based on the IEEE 1394 Standard for High Performance Serial Bus. ${ }^{17}$

2. EIA-762 and EIA-761: These standards ${ }^{24,25}$ define minimum specifications for a one-way data path utilizing an 8 vestigial sideband (VSB) or a 16 VSB remodulator in compliance with ATSC Standard A/53 Annex D. ${ }^{26}$

\# EIA Standards are available at http://www.eia.org for a nominal fee. 
3. EIA-679: This standard ${ }^{27}$ defines a specification for a national renewable security standard (NRSS). It provides an architecture to allow the conditional access functionality to be detached from consumer electronics devices.

4. EIA-770.2 and EIA-770.3 : These standards ${ }^{28,29}$ define the specifications for standard definition and high definition analog component video interfaces, respectively.

Several CEMA working groups have worked on the protection of these interfaces. A summary of this work is given in Table 2. R4 is the Video Systems Committee within CEMA. R4.8, a subcommittee reporting to R4, is responsible for all digital interfaces including their protection. The Joint Engineering Committee (JEC) was formed by CEMA and NCTA $\S \S$ to work on technical issues of common interest.

\begin{tabular}{|l|l|l|}
\hline Working Group & Interface & Work done \\
\hline R4.8 WG2 & IEEE 1394 and RF Remod & $\begin{array}{l}\text { Issued a CFI on November 4, 1998. } \\
\text { Gathered information on 5 proposals: 5C, MRJ } \\
\text { Technology Solutions, NDS, Philips, and } \\
\text { Thomson/Zenith. Published a report }\end{array}$ \\
the proposals.
\end{tabular}

Table 2. Working groups for interface protection

\section{DESIRABLE ATTRIBUTES FOR A COPY PROTECTION SYSTEM}

The first step in developing a system in any field of engineering is to determine the system requirements. Although work on copy protection had been continuing for some time, a list of desirable attributes applicable to a copy protection system was not available until recently. Recognizing the need, CEMA put together the following list:

General

1. Offers a sufficient level of security to "keep honest people honest."

2. Is likely to achieve broad multi-industry consensus and receive support of industries participating in the CPTWG.

Technical

3. Is renewable.

4. Is applicable to one or more of the following four interfaces: IEEE 1394, RF Remod, NRSS A \& B, and Component Video.

5. Has low complexity in implementation, operation, maintenance and administration.

6. Provides transmission and storage protection.

7. Does not result in perceptible degradation of content.

$\S \S$ National Cable Television Association (http://www.ncta.com/). 
8. Does not inhibit desirable and currently available features on CE products such as trick play.

9. Is extendable to general-purpose computing architectures, allowing interoperability of CE and generalpurpose computing devices.

10. Has components available competitively from as large a number of sources as possible.

\section{Consumer}

11. Allows time shifting of transmitted content (i.e., recording) for fair use.

12. Allows place shifting of content (e.g., the ability to play a lawfully made recording at a friend's house on compliant equipment).

13. Allows free copying of content, including over-the-air and non-premium services, accommodates generational control of premium services, and permits the copyright owner to prevent copying of pay-perview and video-on-demand services as well as prerecorded content.

14. Can accommodate changes without impairing the ability of the existing equipment to operate with new content or new equipment to operate with old content.

15. Can include features or accommodate changes without rendering recorded material unviewable to the extent that user has expectation of viewability.

Legal

16. Does not introduce import or export problems for the United States and other major markets.

17. Includes a technological measure which permits legal enforcement against circumvention.

18. Is licensed in accordance with the CEMA Intellectual Property Rights (IPR) policy.

19. Preserves consumer's legal rights of use, including the first sale doctrine.

When the list was completed, it was presented to the CPTWG in March 1999, and later discussed with the MPAA in April 1999. After reviewing the CEMA list, the MPAA published its own list of "attributes of a security environment for distribution of protected high value content." In this list, distributed in May 1999, "Approved" means acceptable to owners of legally protected high value content exercising their individual discretion, for the purpose of protecting lawful rights. It is assumed that all content referenced in the list is legally protected, high value content. The list reflects the views of the individual member companies of the MPAA. All decisions as to whether particular technologies are acceptable, whether to invoke any particular level or form of copy protection, and other matters are for unilateral, independent determination by individual member companies.

MPAA attributes of a security environment for distribution of protected high value content:

1. The following is applicable to all linked transport, display, and recording devices.

2. The same principles apply to CE and IT devices.

3. Digital bit streams are never "in the clear" (i.e., are always encrypted).

4. Bidirectional digital output is allowed only with Approved digital technology protection (e.g., 5C, if Approved).

5. Unidirectional digital output is allowed only with approved digital technology protection (e.g., XCA (see Section 9), if Approved)

6. Standard definition analog video output (NTSC and PAL: 480I, 480P, and 576I lines) must be protected by an Approved Analog Protection System (APS) (e.g., Macrovision) and marked by CGMS-A.

7. All high definition analog video output (greater than 480P, e.g., 720 or 1080 lines) must be protected by an Approved analog protection technique. (For example, a video scrambling technique, yet to be determined and approved. A future system based on watermarking and requiring response under legislation may also be suitable.)

8. All video inputs (digital and analog) must look for and respond to an Approved watermark standard.

9. Licensed devices with recorder function must respond to copy protection flags (CGMS-A, Macrovision, and watermarks). 
10. When only one copy ("copy once") is allowed, such copy must be recorded using an Approved copy protection technology in a manner that does not allow access to the content by non-participating devices and that does not allow further copying.

11. Content providers should be granted express third-party beneficiary rights to enforce licenses.

12. Specific devices should accommodate Approved revocation and renewability mechanisms. Content providers shall have the right to invoke revocation/renewal.

\section{WIPO AND DIGITAL MILLENNIUM COPYRIGHT ACT}

World Intellectual Property Organization (WIPO) is an intergovernmental United Nations organization with headquarters in Geneva, Switzerland. It is responsible for the promotion of the protection of intellectual property throughout the world through cooperation among States, and the administration of various multilateral treaties dealing with the legal and administrative aspects of intellectual property.

Intellectual property comprises two main branches:

- Industrial property: chiefly in inventions, trademarks, industrial designs, and appellations of origin.

- Copyright: chiefly in literary, musical, artistic, photographic and audiovisual works.

The number of member States as of April 15, 1999 was 171. Members include Switzerland, member states of the European Union, USA, China and others.

WIPO Copyright Treaty and WIPO Performances and Phonograms Treaty were adopted by a WIPO conference in Geneva on December 20, 1996 based on existing international treaties (the Berne Convention for the Protection of Literary and Artistic Works as revised in Paris on July 24, 1971, and the Rome Convention for the Protection of Performers, Producers of Phonograms and Broadcasting Organizations of October 26, 1961). Before becoming binding law in the member states, the provisions of the treaties have to be ratified by the member States and national legislation has to be amended. It is not mandatory for WIPO Member States to ratify the treaties; however, the most important Contracting Parties, among them the USA, were expected to do so.

The Digital Millennium Copyright Act (DMCA) ${ }^{32}$ was prepared to amend title 17, United States Code, to implement the WIPO Copyright Treaty and WIPO Performances and Phonograms Treaty, and for other purposes. It includes five titles:

- WIPO Treaties Implementation

- Online Copyright Infringement Liability Limitation

- Computer Maintenance or Repair Copyright Exemption

- Miscellaneous Provisions

- Protection of Certain Original Designs

How the DMCA will be used to enforce copy protection is an open question. The MPAA used the DMCA to sue individuals who attacked the CSS system. With regard to watermarking, the applicability of the DMCA may not be obvious. It is widely believed that if one attempts to deliberately remove or attack a watermark then this a violation of the DMCA. However, does the DMCA require that a watermark has to detected if one is present in the content?

\section{SECURE DIGITAL MUSIC INITIATIVE}

The Secure Digital Music Initiative (SDMI) is a forum that brings together the worldwide recording, consumer electronics and information technology industries to develop specifications for secure distribution of music in digital form. ${ }^{33}$ The mission of SDMI is to enable consumers to conveniently access music, artists and recording companies to protect their intellectual property, and technology and music companies to build successful businesses in their chosen areas. 
SDMI's first achievement is a specification for portable devices. The longer-term project is to complete an overall architecture for delivery of digital music in all forms.

The SDMI Portable Device Specification Part 1 (version 1.0) ${ }^{33}$ contains implementation requirements and reference models for three functional components:

1. Applications: Perform tasks such as content import, library management, playback and rights management.

2. Portable devices and portable media: Store protected content and play it back.

3. Licensed compliant modules: Act as interfaces and translators for communications between applications and portable devices/portable media.

Compliance with the specification is voluntary. It envisioned that the final specification will use a combination of encryption and watermarking. The subsequent parts will describe higher generation portable devices, and a generalized framework for SDMI components.

\section{COPY PROTECTION IN HOME NETWORKS: THE BIGGER PICTURE}

The home network depicted in Figure 6 may receive content from a variety of sources, including cable operators, satellite or terrestrial broadcasters, and telephony centers. Pre-recorded media is also considered to be a content source. A commonality of all these sources is that they protect the content in some private way before delivery. Examples are the protection provided by the DirecTV DSS system and the CSS for DVDs. When the scrambled content reaches the "boundaries" of the network, an authorized "access device" (a DSS set-top box or a DVD player) descrambles the stream, and makes it available for display or storage. The content then has to be sent to a display or storage device.

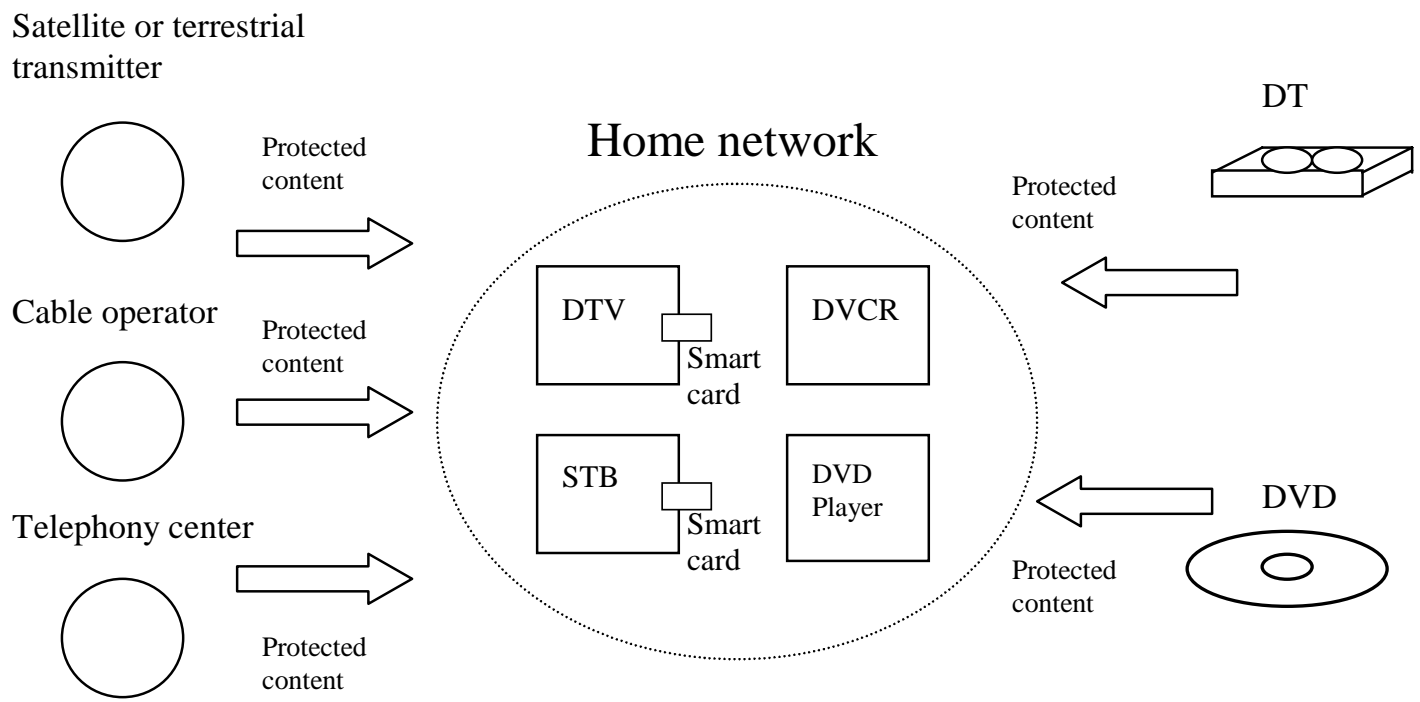

Figure 6. Sources of content for home networks

A global copy protection framework needs to address two problems: Protection of content in transmission and protection of content in storage. Copy protection technologies offer methods and tools to prevent unauthorized access. The approaches used in deploying these technologies can fit into two broad categories: 
1. A collection of separate solutions for transmission and storage.

2. A unified solution that handles both.

The Copy Protection System Architecture (CPSA) presented by IBM, Intel, MEI and Toshiba is an example of the first category. In CPSA, the original secure source is either pre-recorded DVD or electronic distribution. The content, audio or video, is protected by a group of component technologies including CSS (for video), CPPM (for audio), CPRM, 5C, and audio and video watermarking.

A representative example of the second category is the global architecture proposed by Thomson and Zenith. The XCA Copy Protection System Specification defines a system for providing local security of audio and video content during transmission and storage in digital home networks. This task is accomplished by mapping the three basic controls, namely, "playback control", "record control" and "one-generational control" into "viewing control." Under the XCA system, content of economic value is always scrambled - either under the control and responsibility of the distributor or within the confines of the consumer's home network. XCA allows recording of XCA protected content in all conditions. Authorized copies are processed for descrambling and viewing only in licensed devices.

XCA has been developed for use with one-way and two-way digital interfaces. It is primarily a replaceable copy protection system to be used with renewable security devices such as smart cards.

There are three areas of technical compliance in the XCA specification:

- Functional compliance of device elements

- Compliance of bit streams at the NRSS interface

- Compliance of bit streams at the level of XCA Presentation Device interconnection

The XCA Licensing Authority is the entity responsible for administering the copy protection system. An XCA consumer electronics (CE) Device is a device that may perform either or both of the following, optionally in conjunction with a renewable security device:

- Creation of XCA protected bit streams from non-XCA protected programs.

- Descramble portions of XCA protected bit streams

Two XCA CE devices and two removable security devices have been defined with specific functionalities. These normative device types are:

1. Access Device: creates XCA protected content, either alone or in conjunction with a renewable security device.

2. Presentation Device: descrambles XCA protected content, either alone or in conjunction with a renewable security device.

3. Converter Card: a renewable security device that can create XCA protected content from private Conditional Access $(\mathrm{CA})$ protected content.

4. Terminal Card: a renewable security device that can descramble XCA protected content. Its output is compatible with the XCA NRSS interface protection system.

A digital recording device is a device that is able to store or playback XCA protected bit streams, but is unable to create or descramble XCA protected bit streams. Devices that perform digital recording or playback in combination with XCA creation or XCA descrambling shall be classified as an XCA CE Device.

The block diagram in Figure 6 shows the basic XCA system architecture. In principle, XCA concerns itself with the protection of "after" purchase content in the home. The local access and presentation devices are the two essential elements to access, convert and display copyrighted content. The local digital recording device can be used in both CA and XCA domains for storing CA or XCA content. 


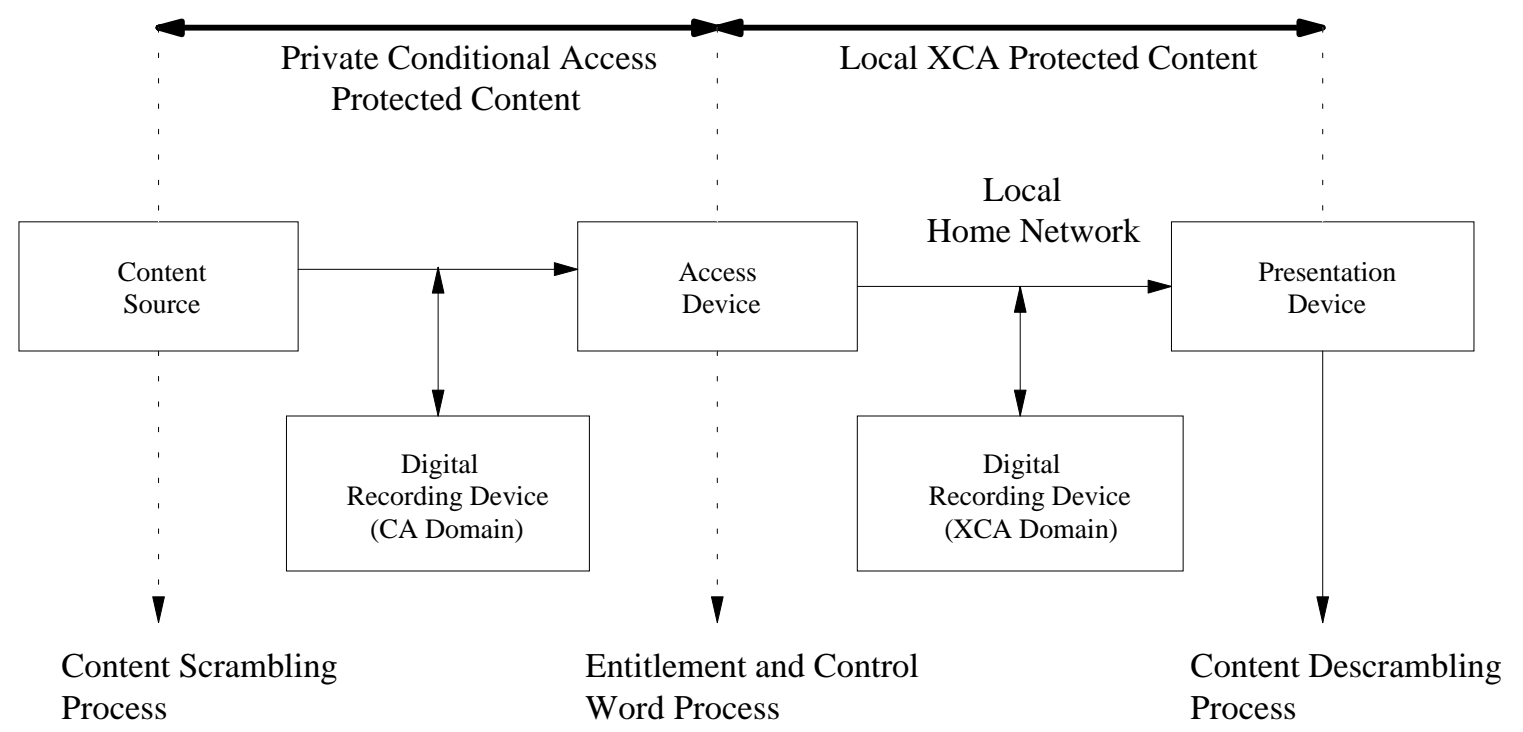

Figure 7. XCA System Model

\section{CONCLUSIONS}

The following conclusions can be drawn from our review of copy protection in consumer electronics devices:

1. Device interoperability is essential: The standard interfaces developed for analog and digital signals will guarantee device interconnectivity in home networks. Nevertheless, some of the systems developed for copy protection are not compatible, and do not provide interoperability. This may present a potential problem for the consumer who may have to know what services are protected by which copy protection systems, and identify the consumer devices supporting those systems.

2. Encryption-based technologies provide "conditional" security: The difficulty in attacking cryptographic tools (ciphers, authentication and digital signature methods) are based on today's computational resources. With the ever increasing power of computing devices, today's secure systems will undoubtedly no longer be robust in the future unless they are upgraded.

3. Watermark-based technologies may require legislation: Watermarking may require legislation with respect to whether the watermark must be detected. In the absence of a law, non-compliant devices in the market place can be used for circumvention. Watermarks may prove useful if implemented as a second line of defense complimentary to encryption.

4. The 3 major industries (CE, IT \& MP) tend to have conflicting requirements: This is an ironical situation. The MPAA expects robust solutions (which are expensive and complex), the CE companies need the least expensive solutions, and the IT industry desires to implement everything in software.

5. Consensus is needed: To reach a common set of goals, the participating industries need to agree on certain legal and technical issues, opening the avenues for progress and closure.

We would like to hope that copy protection will not be a roadblock for successful deployment of digital television. The "digital world" brings many advantages, but also many interesting problems. 


\section{REFERENCES}

D. E. R. Denning, Cryptography and Data Security, Addison-Wesley Publishing Company, 1983.

C. P. Pfleeger, Security in Computing, Prentice-Hall, Inc, 1989.

D. W. Davies and W. L. Price, Security for Computer Networks, John Wiley and Sons, Ltd, 1989.

B. Schneier, Applied Cryptography, John Wiley and Sons, Inc, 1996.

J. Menezes, P. C. van Oorschot, and S. A. Vanstone, Handbook of Applied Cryptography, CRC Press, 1997.

C. E. Shannon, "Communication theory of secrecy systems," Bell Systems Technical Journal, vol. 28, October 1949, pp. 656-715.

7 M.E. Hellman, "An extension of the Shannon theory approach to cryptography," IEEE Transactions on Information Theory, vol. 23, no. 5, May 1977, pp. 289-294.

8 W. Diffie and M.E. Hellman, "New directions in cryptography," IEEE Transactions on Information Theory, vol. 22, no.6, November 1976, pp. 644-654.

9 W. Diffie and M.E. Hellman, "Privacy and authentication: an introduction to cryptography," Proceedings of the IEEE, vol. 67, no. 3, March 1979, pp. 397-427.

10 M. D. Swanson, M. Kobayashi, and A. H. Tewfik, "Multimedia data-embedding and watermarking technologies," Proceedings of the IEEE, vol. 86, no. 6, June 1998, pp. 1064-1088.

11 F. A. P. Petitcolas, R. J. Anderson, and M. G. Kuhn, "Information hiding - a survey," Proceedings of the IEEE, vol. 87, no. 7, July 1999, pp. 1062-1078.

12 F. Hartung and M. Kutter, "Multimedia watermarking techniques," Proceedings of the IEEE, Vol. 87, No. 7, July 1999, pp. 1079-1107.

13 R. B. Wolfgang, C. I. Podilchuk, and E. J. Delp, "Perceptual watermarks for digital images and video," Proceedings of the IEEE, vol. 87, no. 7, July 1999, pp. 1108-1126.

14 I. Cox, J. Kilian, F. T. Leighton, and T. Shamoon, "Secure spread spectrum watermarking for multimedia," IEEE Transactions on Image Processing, vol. 6, no. 12, December 1997, pp. 1673-1687.

15 A. Bell, "The dynamic digital disk," IEEE Spectrum, vol. 36, no.10, October 1999, pp.28-35.

16 J. A. Bloom, I. J. Cox, T. Kalker, J-P. M. G. Linnartz, M. L. Miller, and C. B. S. Traw, "Copy protection for DVD video," Proceedings of the IEEE, vol. 87, No. 7, July 1999, pp. 1267-1276.

17 I. J. Wickelgren, "Facts about FireWire," IEEE Spectrum, vol. 34, no. 4, April 1997. p 19-25.

18 "Review and Findings of Submitted Proposals," Digital Transmission Discussion Group, Version 1.0, November 11, 1997.

19 "5C Digital Transmission Content Protection," available at http://www.dtcp.com/.

20 “Interim Report, Results of Phases I and II," Data Hiding Subgroup, Version 0.15, April 16, 1998, (available at http://www.dvcc.com/dhsg).

21 A. Pressman, "Hollywood sues sites over DVD software," Reuters, January 14, 2000.

22 "ISO 7816-1 - Identification cards - integrated circuits cards with contacts," ISO 1987.

23 “EIA-775 DTV 1394 Interface Specification,” December 1998.

24 "EIA-761 DTV Re-modulator Specification with Enhanced OSD Capability,” November 1998.

25 "EIA-762 DTV Re-modulator Specification," August 1998.

26 "Advanced Television Standards Committee (ATSC) Standard A/53," available at http://www.atsc.org.

27 "EIA-679B National Renewable Security Standard," September 1998.

28 "EIA 770.2 Standard Definition TV Analog Component Video Interface," September 1998.

29 "EIA 770.3 High Definition TV Analog Component Video Interface," September 1998.

30 "Review of Information Submitted in Response to the CFI," R4.8 Working Group 2, July 30, 1999.

31 "Compilation of Responses to Further CFI on DTV Analog Component Video Interface," available at http://www.cemacity.org/works/pubs.

32 "Digital Millennium Copyright Act," available at http://lcweb.loc.gov/copyright.

33 "Guide to SDMI Portable Device Specification," available at http://www.sdmi.org. 